\title{
DESENVOLVIMENTO E VALIDAÇÃO DE METODOLOGIA ANALÍTICA PARA DETERMINAÇÃO DE ITRACONAZOL EM PRODUTOS FARMACÊUTICOS POR CLAE
} Edith Cristina Laignier Cazedey, Roberta de Cássia Pimentel Azevedo, Érika de Fátima Silva e Magali Benjamim de Araújo*
Departamento de Farmácia, Universidade Federal de Alfenas, Rua Gabriel Monteiro da Silva, 714, 37130-000 Alfenas - MG, Brasil

Recebido em 3/1/06; aceito em 28/9/06; publicado na web em 14/5/07

\begin{abstract}
DEVELOPMENT AND VALIDATION OF THE METHODOLOGY FOR EVALUATION OF ITRACONAZOLE IN PHARMACEUTICAL PRODUCTS BY HPLC. Itraconazole is a synthetic antifungal drug administered orally with a broad spectrum of activity against mycotic infections. The present work consists of the development and validation of analytical methodology for evaluation of itraconazole in pharmaceutical products by high performance liquid chromatography. The separation was made using the reversed-phase column LC-18, acetonitrile/diethylamine $0.05 \% \mathrm{v} / \mathrm{v}, 60: 40 \mathrm{v} / \mathrm{v}, \mathrm{pH} 8.0$ as mobile phase, methanol as solvent and detection and quantification at $254 \mathrm{~nm}$. The results here obtained show that the analytical methodology is accurate, reproducible, robust and linear over the concentration range $8.0-12.0 \mu \mathrm{g} / \mathrm{mL}$ of itraconazole. The method was applied to pharmaceutical capsules containg itraconazole pellets and showed to be efficient, yielding good results.
\end{abstract}

Keywords: itraconazole; validation; HPLC.

\section{INTRODUÇÃO}

O itraconazol (Figura 1) é um fármaco antifúngico com característica química de um bis-triazólico resultante da substituição do anel imidazólico por triazólico, o que favoreceu amplo espectro de ação e seletividade para o citocromo $\mathrm{P}_{450}$ da célula fúngica ${ }^{1,2}$.

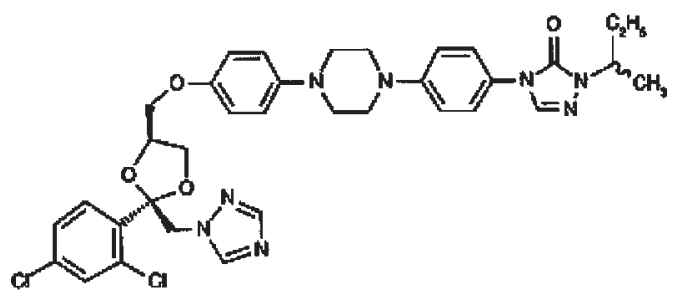

Figura 1. Estrutura química do itraconazol

Apresenta amplo espectro de ação sendo empregado no tratamento de micoses sistêmicas, como aspergilose, blastomicose, candidíase, cromomicose, esporotricose, histoplasmose e paracoccidioidomicose. Nas micoses superficiais é empregado no tratamento de candidíase oral, candidíase vulvovaginal, ceratite micótica, dermatofitoses, onicomicoses, pitiríase versicolor, Tinea cruris, Tinea manuum e Tinea pedis ${ }^{3,4}$.

O mecanismo de ação baseia-se na capacidade de inibir a síntese do ergosterol, um componente vital da membrana da célula dos fungos. A conseqüência do bloqueio da síntese do ergosterol é um aumento da permeabilidade da membrana celular, desencadeando alterações morfológicas que resultam em necrose celular ${ }^{2,3,5}$. O itraconazol é altamente lipofílico ( $\mathrm{pKa}=3,7$ ), apresentando baixa solubilidade em soluções aquosas e rápida absorção oral ${ }^{6}$.

Em virtude de suas propriedades farmacológicas, este fármaco vem sendo bastante empregado na terapêutica das micoses superficiais e sistêmicas. A literatura registra inúmeros trabalhos referentes ao itraconazol e sua determinação simultânea com fluconazol, voriconazol em fluidos biológicos, empregando a cromatografia líquida de alta eficiência com detecção no ultravioleta e, também, com detector de massas ${ }^{7-10}$.

*e-mail: magali@unifal-mg.edu.br
A monografia do produto farmacêutico não se encontra inscrita, até o momento, em compêndios oficiais. Diante disto, faz-se necessário o desenvolvimento e a validação de metodologia analítica capaz de quantificar o fármaco nas especialidades farmacêuticas comercializadas no mercado nacional.

\section{PARTE EXPERIMENTAL}

\section{Materiais}

Produtos farmacêuticos, padrão de referência e solventes

O Itraconazol Sigma (Alemanha), lote 113K1501, foi utilizado como substância química de referência, apresentando validade até 01/06.

A forma farmacêutica -cápsulas contendo itraconazol $100 \mathrm{mg}$ em pellets, lote 0301710 , validade 11/05 (Amostra 1)- foi cedida pela Aché Laboratórios e o Itraconazol $100 \mathrm{mg}$-em pellets (Amostras 2 e 3)- foi obtido em farmácia de manipulação.

Os reagentes empregados foram ácido acético (Vetec, Brasil) grau UV/HPLC; acetonitrila (Vetec, Brasil) grau UV/HPLC; dietilamina (Vetec, Brasil) grau analítico e metanol (Vetec, Brasil) grau UV/HPLC.

\section{Equipamentos}

Foi utilizado cromatógrafo a líquido Shimadzu (Japão), série LC10A com sistema de integração CLASS-VP 5.02, bomba LC-AVP, degaseificador DGU-14 A, injetor manual 7725i com alça de amostragem de $20 \mu \mathrm{L}$ (Rheodyne, EUA), detector SPD-10AVP, válvula FCV-10ALVP, forno de coluna CTO-10AVP e controlador SCL$10 \mathrm{AV}$. As colunas cromatográficas foram a Supelcosil ${ }^{\circledR}$ LC-18 (Supelco, EUA) com $10 \mathrm{~cm}$ de dimensão, 4,6 mm de d.i., $5 \mu \mathrm{m}$ de tamanho de partícula, código 15083-008 (coluna 1), Hypersil BDS ${ }^{\circledast}$ C-18 (Agilent Technologies, EUA), $10 \mathrm{~cm}$ de dimensão, 4,6 mm de d.i., $3 \mu \mathrm{m}$ de tamanho de partícula, código 79916BD-354 (coluna 2) e pré-coluna Superguard ${ }^{\circledR}$ LC-18 (Supelco, EUA) com $2 \mathrm{~cm}$ de dimensão, 4,6 mm de d.i. e $5 \mu \mathrm{m}$ de tamanho de partícula. Também foram utilizados: aparelho de ultrasson modelo USC2800A (Unique, 
Brasil); balança analítica modelo 410 (Kern, Alemanha); bomba à vácuo (Nova Técnica, Brasil); medidor pH modelo PA 200 (Marconi, Brasil); Sistema de filtração a vácuo (Millipore, Brasil); membrana PTFE de 0,45 $\mu \mathrm{m}$ e $13 \mathrm{~mm}$ de diâmetro (Millipore, Brasil) e sistema de purificação de água TKA modelo LAB-UPW (Carl Zeiss Jena, Alemanha).

\section{Metodologia}

\section{Parâmetros cromatográficos}

A escolha da fase móvel foi realizada através da mistura de solventes constituídos por acetonitrila e dietilamina 0,05\% v/v (60:40 $\mathrm{v} / \mathrm{v}), \mathrm{pH} 8,0$ e utilizando metanol para solubilizar as amostras. As soluções das amostras e de estoque foram preparadas no momento da sua utilização. As condições de operação foram vazão de $1 \mathrm{~mL}$ $\min ^{-1}$, tempo de corrida de 6 min e detecção no UV em $254 \mathrm{~nm}$.

\section{Validação da metodologia analítica $a^{11-15}$}

Para estudo da linearidade preparou-se solução da substância química de referência, na concentração de $100 \mu \mathrm{g} / \mathrm{mL}$, dissolvendose o itraconazol em metanol. Alíquotas de 0,$8 ; 0,9 ; 1,0 ; 1,1$ e $1,2 \mathrm{~mL}$ foram transferidas para balões volumétricos de $10 \mathrm{~mL}$, obtendo-se soluções com concentrações variando de 8,0 a $12,0 \mu \mathrm{g} / \mathrm{mL}$. As soluções de itraconazol, substância química de referência, foram preparadas em quatro réplicas, filtradas em filtros de $0,45 \mu \mathrm{m}$ e procedendo-se às injeções das soluções em cromatógrafo a líquido. A curva de calibração foi construída plotando-se os valores médios das áreas dos picos cromatográficos em função das concentrações.

A especificidade do método foi determinada preparando-se soluções da substância química de referência e da forma farmacêutica cápsula (Amostra 1) nas concentrações finais de $10,0 \mu \mathrm{g} / \mathrm{mL}$ de itraconazol, utilizando-se metanol como solvente. As soluções de padrão e amostra, previamente filtradas em filtros de $0,45 \mu \mathrm{m}$, foram injetadas em cromatógrafo, registrando-se os valores das áreas dos picos cromatográficos, calculando-se o percentual de resposta para o método e a estimativa de desvio padrão da determinação.

A precisão do método foi avaliada pela repetibilidade intradias e intermediária. Na precisão intradias prepararam-se 12 réplicas das soluções de itraconazol substância química de referência, utilizando-se metanol como solvente, nas concentrações finais de 10 $\mu \mathrm{g} / \mathrm{mL}$. Procedeu-se às injeções das réplicas das soluções, previamente filtradas em filtros de $0,45 \mu \mathrm{m}$, no mesmo dia, mas em corridas diferentes, registrando-se os valores das áreas dos picos cromatográficos e calculando-se a estimativa do desvio padrão das determinações.

Para a precisão intermediária, avaliada através da comparação entre as injeções de uma mesma concentração realizadas em dias diferentes e por analistas diferentes, prepararm-se 6 réplicas de soluções de itraconazol substância química de referência em cada dia, utilizando-se metanol como solvente, nas concentrações finais de $10 \mu \mathrm{g} / \mathrm{mL}$. Procedeu-se às injeções das soluções, previamente filtradas em filtros de $0,45 \mu \mathrm{m}$, registrando-se os valores das áreas dos picos cromatográficos e calculando-se a estimativa do desvio padrão das determinações.

A exatidão do método foi avaliada pelo teste de recuperação onde foram preparadas, em triplicata, soluções da forma farmacêutica cápsulas (Amostra 1) e da substância química de referência nas concentrações finais de $5,0 \mu \mathrm{g} / \mathrm{mL}$, utilizando-se metanol como solvente. Volumes apropriados da solução padrão foram utilizados para obtenção de três níveis de concentração na amostra, a saber 8,0; 10,0 e $12,0 \mu \mathrm{g} / \mathrm{mL}$. As soluções foram analisadas utilizando-se o método proposto. Foram feitas três determinações para cada concentração e calculadas as percentagens de recuperação (\% R).
A robustez do método foi avaliada mediante o preparo de réplicas de solução de itraconazol substância química de referência, em metanol, nas concentrações finais de $10,0 \mu \mathrm{g} / \mathrm{mL}$ que, após terem sido filtradas em filtros de $0,45 \mu \mathrm{m}$, foram injetadas no cromatógrafo utilizando-se colunas cromatográficas diferentes (colunas 1 e 2) e variando-se o $\mathrm{pH}$ da fase móvel em -0,2 unidades ( $\mathrm{pH} 7,8)$.

\section{Doseamento das amostras de cápsulas}

Foram preparadas, em 6 réplicas, soluções de itraconazol substância química de referência e das amostras das cápsulas 1, 2 e 3, em metanol, nas concentrações finais de $10 \mu \mathrm{g} / \mathrm{mL}$. As soluções de padrão e das amostras, previamente filtradas em filtros de 0,45 $\mu \mathrm{m}$, foram injetadas em cromatógrafo, registrando-se os valores das áreas dos picos cromatográficos e calculando-se o teor de itraconazol presente nas amostras.

\section{RESULTADOS E DISCUSSÃO}

Trabalho anterior ${ }^{16}$ relata a utilização da fase móvel constituída de acetonitrila e água contendo dietilamina $0,05 \%$ v/v $(60: 40 \mathrm{v} / \mathrm{v})$ com $\mathrm{pH}$ ajustado em 8,0 com ácido acético $30 \%$, utilizando vazão de $1,2 \mathrm{~mL} / \mathrm{min}$, coluna Microsorb -MV ${ }^{\circledR} \mathrm{C} 18$ de $25 \mathrm{~cm}$ x $5 \mu \mathrm{m}$ e detecção em $254 \mathrm{~nm}$ para estudos de dissolução e biodisponibilidade de itraconazol. A fase móvel foi otimizada envolvendo estudos relacionados com a alteração das proporções dos solventes utilizados e variação do $\mathrm{pH}$ de forma proporcionar a redução do tempo de retenção, favorecendo análise rápida para ser aplicada na rotina. Foram fixados os seguintes parâmetros: fase móvel constituída de acetonitrila e dietilamina $0,05 \% \mathrm{v} / \mathrm{v}(60: 40 \mathrm{v} / \mathrm{v}), \mathrm{pH}$ 8,0 e metanol como solvente das amostras, vazão de $1 \mathrm{~mL} / \mathrm{min}$, tempo de corrida 6 min e detecção em $254 \mathrm{~nm}$ (Figura 2). A fase estacionária utilizada tem na sua constituição sílica e, embora o pH da fase móvel seja básico (pH 8), a recomendação da literatura é que colunas à base de sílica não sejam utilizadas acima deste $\mathrm{pH}^{17}$.

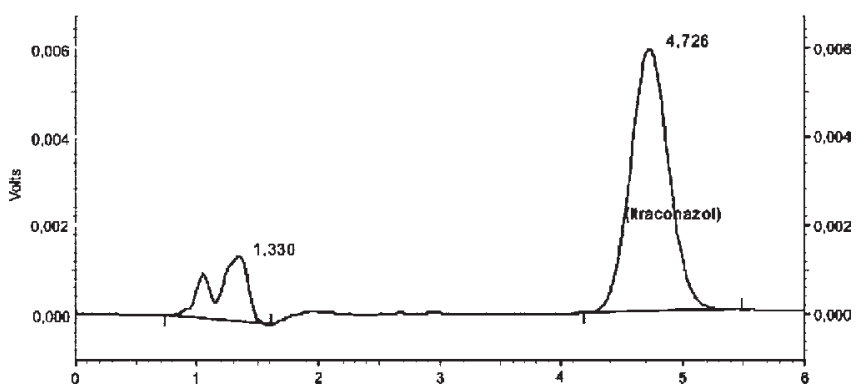

Figura 2. Perfil cromatográfico de itraconazol na concentração de $10 \mu \mathrm{g} /$ $m L$, utilizando como fase móvel acetonitrila e dietilamina 0,05\% v/v (60:40 $v / v$ ), pH 8,0, com detecção em 254 nm (número de pratos teóricos= 1000; fator de assimetria 1,03; fator de capacidade 5,30)

Tabela 1. Valores médios de áreas de pico e da estimativa do desvio padrão obtidos para itraconazol substância química de referência, utilizando-se como fase móvel acetonitrila e dietilamina $0,05 \% \mathrm{v} /$ $\mathrm{v}(60: 40 \mathrm{v} / \mathrm{v}), \mathrm{pH} 8,0$ a $254 \mathrm{~nm}$

\begin{tabular}{lcc}
\hline $\begin{array}{l}\text { Concentração } \\
(\mu \mathrm{g} / \mathrm{mL})\end{array}$ & Média das áreas & $\begin{array}{c}\text { Estimativa do } \\
\text { desvio padrão }(\%)\end{array}$ \\
\hline 8,0 & 213474 & 0,75 \\
9,0 & 240975 & 0,98 \\
10,0 & 269478 & 0,56 \\
11,0 & 297762 & 0,21 \\
12,0 & 329496 & 0,63 \\
DPR $(\%)$ médio & & 0,63
\end{tabular}


Tabela 2. Valores obtidos na determinação dos parâmetros de validação do método para itraconazol, utilizando como fase móvel acetonitrila e dietilamina $0,05 \% \mathrm{v} / \mathrm{v}(60: 40 \mathrm{v} / \mathrm{v}), \mathrm{pH}=8,0$ a $254 \mathrm{~nm}$

\begin{tabular}{|c|c|c|c|c|c|}
\hline Parâmetros & Condições & $\begin{array}{c}\text { Níveis de } \\
\text { fortificação }(\mu \mathrm{g} / \mathrm{mL})\end{array}$ & $\begin{array}{l}\text { Média } \\
\text { das áreas }\end{array}$ & $\begin{array}{c}\text { Estimativa do } \\
\text { desvio padrão (\%) }\end{array}$ & $\begin{array}{c}\text { Valores de } \\
\text { Recuperação (\%) }\end{array}$ \\
\hline \multirow[t]{2}{*}{ Especificidade } & Sem excipientes & & 271079 & $0,67(n=6)$ & \\
\hline & Com excipientes & & 270609 & $0,76(n=6)$ & \\
\hline \multirow[t]{2}{*}{ Precisão intradias } & Corrida 1 & & 268058 & $0,98(n=6)$ & \\
\hline & Corrida 2 & & 246733 & $0,83(n=6)$ & \\
\hline \multirow[t]{2}{*}{ Precisão intermediária } & $1^{\mathrm{o}} \mathrm{dia}$ & & 248516 & $0,23(n=6)$ & \\
\hline & $2^{\circ}$ dia & & 244951 & $0,46(n=6)$ & \\
\hline \multirow[t]{3}{*}{ Exatidão } & Adição de & 8,0 & 204210 & $1,20 \quad(n=3)$ & 101,00 \\
\hline & padrão & 10,0 & 254887 & $0,31 \quad(n=3)$ & 101,20 \\
\hline & & 12,0 & 305830 & $1,31 \quad(n=3)$ & 101,60 \\
\hline
\end{tabular}

Pelos parâmetros apresentados na Tabela 1, é possível observar que a linearidade do método, obtida na faixa de concentração de 8,0 a $12,0 \mu \mathrm{g} / \mathrm{mL}$, apresenta coeficiente de correlação $\mathrm{R}^{2}=0,9993$ $(\mathrm{y}=28883 \mathrm{x}-18594)$, com estimativa de desvio padrão entre as determinações menor que $2 \%$, demonstrando haver correlação linear entre as concentrações de itraconazol e as áreas de pico no tempo de retenção.

A especificidade do método, parâmetro importante na validação de um método cromatográfico, foi avaliada pelo percentual de resposta de $99,80 \%$ com estimativa de desvio padrão de $0,67 \%$ para o fármaco sem os excipientes e $0,76 \%$ para o fármaco contaminado com os excipientes, permitindo comprovar que os pellets da formulação não interferem na determinação quantitativa do itraconazol.

A precisão do método, sob condições fixadas, foi determinada pela estimativa do desvio padrão, por aplicação do método à amostra em condições idênticas. Os valores médios da estimativa de desvio padrão de 0,90 e $0,34 \%$ demonstraram que o método possui boa precisão quando analisado no mesmo dia e em dias diferentes. São aceitáveis valores de desvio padrão relativo igual ou abaixo de $5 \%{ }^{12}$.

A exatidão foi realizada pela técnica de adição de padrão e avaliada pelo teste de recuperação, onde quantidades conhecidas da substância química de referência foram adicionadas ao medicamento. Os valores médios de recuperação de 101,30\% (amostra 1) indicam recuperação adequada. Valores de percentagem de recuperação entre $98 \%$ e $102 \%$ são aceitáveis ${ }^{11,13}$.

A robustez de um método analítico, que é a medida de sua capacidade em resistir a pequenas variações dos parâmetros analíticos, foi verificada ao se substituir a coluna cromatográfica e alterar o $\mathrm{pH}$ da fase móvel para 7,8. Com os dados obtidos foi realizada a análise de variância, constatando-se que o método não sofre variação com as alterações propostas. Os valores obtidos não são significativamente diferentes em nível de $95 \%$ de confiança ${ }^{11,12,15}$. A Tabela 2 apresenta os parâmetros de validação da metodologia analítica.

$\mathrm{O}$ método analítico proposto foi aplicado à forma farmacêutica cápsulas das amostras 1, 2 e 3 (Tabela 3). As médias dos teores obtidos para a quantificação do itraconazol nos pellets, de 20,80;

Tabela 3. Valores obtidos no doseamento das amostras 1,2 e 3 de itraconazol, utilizando como fase móvel acetonitrila e dietilamina $0,05 \% \mathrm{v} / \mathrm{v}(60: 40 \mathrm{v} / \mathrm{v}), \mathrm{pH} 8,0$ a $254 \mathrm{~nm}$

\begin{tabular}{lcccc}
\hline & Padrão & Amostra 1 & Amostra 2 & Amostra 3 \\
\hline Média das Áreas & 269572 & 254912 & 267477 & 271080 \\
DPR(\%) & 0,35 & 0,44 & 0,52 & 067 \\
Teor(\%) & 22,00 & 20,80 & 21,83 & 22,12 \\
\hline
\end{tabular}

21,83 e $22,12 \%$, com estimativas de desvio padrão de 0,$44 ; 0,52$ e $0,67 \%$ para as amostras 1,2 e 3 , respectivamente, estão dentro dos limites da especificação farmacêutica que foi fornecida pelo fabricante que é de 19,80 a $24,20 \%$ de itraconazol nos pellets.

\section{CONCLUSÃO}

O método proposto mostrou-se eficiente e confiável, apresentando linearidade nas faixas de 8,0 a $12,0 \mu \mathrm{g} / \mathrm{mL}$, repetibilidade, exatidão e robustez permitindo o uso em análises rotineiras do itraconazol nas especialidades farmacêuticas comercializadas no mercado nacional e nos produtos manipulados.

\section{AGRADECIMENTOS}

À Aché Laboratórios pelo fornecimento da amostra 1 de itraconazol e à FAPEMIG pelo suporte financeiro do projeto.

\section{REFERÊNCIAS}

1. Janssen, P. A. J.; Vanden Bossche, H.; Arch. Pharm. Chem. Sci. 1987, 15, 23.

2. Korolkovas, A.; Dicionário Terapêutico Guanabara, ed. 2005/2006, Guanabara Koogan: Rio de Janeiro, 2006, p. 18.9.

3. Lacaz, C. S.; Del Nero, G. Em Farmacologia; Silva, P., ed.; 6 ${ }^{\mathrm{a}}$ ed.; Guanabara Koogan: Rio de Janeiro, 2002, p. 1118.

4. Machado, A. R. L. Em Farmacologia Clínica; Fuchs, F. D.; Wannmacher, L. eds.; 2a ed.; Guanabara Koogan: Rio de Janeiro, 1998, p. 282.

5. Viviane, M. A.; Chemotherapy 1992, 28, 35.

6. Heykants, J.; Michiels, M.; Meuldermans, W.; Monabaliu, J.; Lavrisjen, K.; van Peer, A.; Levron, J. C.; Woestenborghs, R.; Gauwenbergh, G.; Prous Science Publishers 1987, 223.

7. Khoschsorur, G.; Fruehwirth, F.; Zelzer, S.; Antimicrob. Agents Chemother. $2005,49,3569$.

8. Choi, Y. W.; Nam, D.; Kang, K. H.; Ha, K.W.; Han, I. H.; Chang, B. K.; Yoon, M.; Lee, J.; Bull. Kor. Chem. Soc. 2006, 27, 291.

9. Gal, J.; Pyrgaki, C.; Bannister, S. J.; Gera, L.; Gerber, J. G.; Ther. Drug Monit. 2005, 27, 219.

10. Gutteck-Amsler, U.; Rentsch, K. M.; Ther. Drug Monit. 2005, 27, 241

11. Barros, C. B.; Hirata, Y. S.; Validação de Métodos Analíticos, 1999, p. 24.

12. Brasil. Resolução RE nº99 de 29/5/2003; Diário Oficial da União, Brasília, DF, 02/06/2003, seção 1 - Agência Nacional de Vigilância Sanitária aprova o Guia para validação de métodos analíticos e bioanalíticos.

13. Guia para Laboratórios Químicos; Um Auxílio à Organização e ao Credenciamento/ INMETRO, Interciência Ltda: Rio de Janeiro, 2000, p. 41.

14. International Conference on Harmonisation (ICH); Topic Q2B - Validation of Analytical Procedures: Methodology, Proceedings of the Commission of the European Communities, 1996.

15. United States Pharmacopoeia; $29^{\text {th }}$ ed.; United States Pharmacopoeia Convention: Rockville, 2006, p. 3050.

16. Kapsi, S. G.; Ayres, J. W.; Int. J. Pharm. 2001, 229, 193.

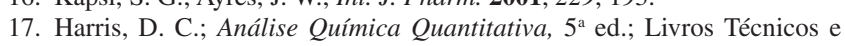
Científicos: Rio de Janeiro, 2001, p. 616. 